puberty (PPP), advanced puberty and rapidly progressive puberty. Exclusion criteria: not going to follow-up visits. Statistical analysis by SPSS 23 .

Results A total of 311 subjects (14 men, 297 women) who were referred by their pediatricians, attended the first visit to the endocrinology department with an average age of 7.8 years (95\% CI: 7.7-8). The distribution of subjects was: involuted precocious breast development $(n=25)$, non-progressive precocious breast development $(\mathrm{n}=19)$, PPC $(\mathrm{n}=156)$, PPP $(\mathrm{n}=3)$, advanced puberty $(\mathrm{n}=88)$ and rapidly progressive puberty $(n=20)$. Male subjects who were diagnosed of precocious puberty had at first visit a mean age of 8.7 years (95\% CI: 7.8-9.5), and women had a mean age of 7.7 years (95\% CI: 7.5-8). There are significant differences regarding bone age at diagnosis (more advanced in central precocious puberty and rapidly progressive puberty). There is a statistically significant association between precocious puberty and the fact of being adopted (X2: 11.262; p: 0.046). The LH at 3 hours of the GnRH test was significantly higher in the PPC group (mean value of LH 14UI/L, IC95\%: 11.5-16.6) compared to the others. Patients were identified who did not meet the classic criteria of the diagnosis of PPC in the Procrin test, however they presented clinical characteristics in the follow-up that led to the diagnosis. MRI: significant association between having MRI findings and presenting PPC (X2: 38.262; p: 0. 000), however incidental findings where found in MRI of patients with advanced puberty. A total of $69 \%$ of patients with PPC and $17.6 \%$ patients with advanced puberty (including here the rapidly progressive forms) received treatment.

Conclusions We present the data of a population of children of both sexes with clinical manifestations suggestive of precocious/advanced puberty evaluated and followed between the years 2010 and 2018. The results coincide with those described in previous studies.

\section{P296 GENU VALGUM AS A RARE CLINICAL MANIFESTATION IN CHILD WITH PRIMARY HYPERPARATHYROIDISM: A CASE REPORT}

Yaulia Yanrismet*, Bambang Tridjaja. Departement of Child Health, Faculty of Medicine University of Indonesia, Jakarta, Indonesia

\subsection{6/archdischild-2019-epa.646}

Background Primary hyperparathyroidism (PHPT) is a rare case in children with the incidence of $2-5: 100,000$. Its manifestations vary greatly from asymptomatic to severe complications. Genu valgum is a scarce clinical manifestation of PHPT in children and often misdiagnosed with rickets. There were scanty case reports about genu valgum in children with PHPT worldwide. It is generally sporadic and nearly $65 \%$ caused by adenomas; curable by surgical therapy. Delayed in diagnosis increases the morbidity and mortality rate

Objectives To report a rare case and its scarce clinical manifestation in children

Case presentation A 13-year-old boy with bilateral genu valgum was referred with rickets. He also had other clinical feature of skeletal abnormalities such as progressive difficulty in walking, bone and joint pain and muscle weakness since six months prior to admission. He was normal at birth. Formerly he was diagnosed as rickets and had calcium and vitamin D therapy, but no improvement. He, thereafter, referred to the National referral hospital CiptoMangunkusumo hospital. Skeletal survey revealed decreased bone density by epiphyseal plate widening, bilateral lateral clavicle tip, sacroiliac joint bilateral, and symphysis pubic resorption with rugger-jersey spine, slipped capital femoral epiphysis bilateral. Metabolic profile showed low vitamin D level with hypercalcemia and mild hypophosphatemia. Intact parathyroid level was very high (1301 pg/ml). Bone mineral density below the value of children in his age (whole body z-score was -5,5 and AP spine zscore was -4,5). Kidney function test and liver function test were normal. MSCT of the larynx showed solid lesions with the contrast in the right thyroid gland inferoposterior pole as high as vertebrae cervical 6 to thoracic 1, confirmed with dual time Tc99 parathyroid scintigraphy with the appearance of pathological activity at the inferior of the right thyroid suspected as parathyroid adenoma. He underwent parathyroidectomy surgery; the results of anatomical pathology examination in accordance with adenomas. Post surgery he experienced hungry bone syndrome.

Conclusion Genu valgum is a scarce clinical manifestation in children with PHPT which mostly wrongly diagnosed and treated as rickets. This condition occurs in PHPT children at pubertal aged with unknown mechanism. The misstep of making the diagnosis will lead to higher morbidity and mortality. Therefore, early diagnosis in children with genu valgum who suffer PHPT would prevent further bone damage and irreversible complications. A panel of test which includes parathyroid serum examination is warranted in metabolic bone disease.

Genu valgum, primary hyperparathyroidism, case report

\section{P297 CONSTANT UNCERTAINTY: MOTHERS' EXPERIENCES OF CARING FOR CHILDREN RECEIVING GROWTH HORMONE TREATMENT (GHT)}

${ }^{1,2}$ Rasha Alsaigh*, ${ }^{2}$ Imelda Coyne. ${ }^{1}$ King Abdulaziz University, College of Nursing, Jeddah, Saudi Arabia; ${ }^{2}$ Trinity College Dublin, School of Nursing and Midwifery, Dublin , Ireland

\subsection{6/archdischild-2019-epa.647}

Uncertainty has been noted as a significant feature of parental experiences of childhood chronic illnesses needing medical treatments in general and growth hormone treatment (GHT) in particular. However, there is insufficient research exploring the extent of which uncertainty features in the experiences of parents caring for children receiving GHT or the dimensions, which their uncertainty may take. Drawn from 16 in depth interviews and eight diaries from 16 mothers from the Republic of Ireland who had children with a growth disorder requiring daily administration of GHT, this paper highlights their experiences of uncertainty when it comes to dealing with GHT. It was concluded that the dimensions of uncertainty experienced by the mothers were: diagnostic, treatment, future and social stigma uncertainty. It also demonstrates the significant challenges of coping with these uncertainties. By recognising the dimensions of uncertainty faced by mothers caring for children receiving GHT, health care professionals can anticipate these uncertainties and help minimise some of the stress and anxiety associated with them. 